\title{
The effect of thermal vibrations on extended $x$-ray absorption fine structure. $I$
}

\author{
John J. Boland and John D. Baldeschwieler \\ Division of Chemistry and Chemical Engineering, California Institute of Technology, Pasadena, California \\ 91125
}

(Received 18 April 1983; accepted 21 December 1983)

\begin{abstract}
The form of the Debye-Waller factor in EXAFS is discussed in detail, and an expression is obtained for this factor in a general three-atom system of $C_{s}$ symmetry. The normal modes which contribute to the Debye-Waller factors for each scattering path are dependent on the symmetry of the system. A series of model three-atom systems with $C_{2 v}$ symmetry are studied and the DebyeWaller factors as a function of the bridging angle are discussed for each of these systems. The temperature dependence of the Debye-Waller factor for each scattering path is also studied. In a system of $C_{2 v}$ symmetry, approximating the double and triple scattering Debye-Waller factors by the second shell single scattering factor is shown to be strictly valid only for a system close to linearity. The error due to this approximation is dependent upon the amplitude of the individual scattering paths and is shown to increase with temperature. When the single scattering contribution is unimportant, there is shown to exist a temperature where the above approximation is exact.
\end{abstract}

\section{INTRODUCTION}

In recent years extended $\mathrm{x}$-ray absorption fine structure (EXAFS) spectroscopy has been used extensively as a structural tool. Although the existence of the extended structure has been known for many years, it was not until Sayers, Stern, and Lytle ${ }^{1}$ introduced a simple parametrization of the structure that the informational content of EXAFS was realized. In addition, the development of synchrotron and laboratory EXAFS facilities has made the technique available to a large number of investigators and provided a major impetus towards the development of EXAFS as a structural tool.

Much of the present interest in EXAFS stems from its short range nature which allows the technique to be applied in instances where other structural tools are not suitable. The sensitivity to short range order insures that the EXAFS contributions from distant shells are small, which in turn, greatly simplifies an analysis of the extended fine structure. The ability to change the $x$-ray frequency over a large energy range allows structural information to be obtained on the local environment about each absorbing atom in the sample. In principle, bond distances, coordination numbers, and the types of neighboring atoms present may be determined from an EXAFS study. ${ }^{2}$

The purpose of this paper is to provide a detailed study of the nature of the Debye-Waller factor in EXAFS. In general, the Debye-Waller factor has components due to static and vibrational disorder. This paper will address the problem of thermally excited vibrations and their effect on EXAFS spectra. Previous studies of the Debye-Waller factor in EXAFS were concerned solely with single scattering events. $^{3-6}$ Recently, however, there has been considerable interest in multiple scattering EXAFS processes and the determination of bond angles from an analysis of the extended structure. ${ }^{7,8}$ The possibility of determining accurate bond angles from an EXAFS study is obviously dependent on an understanding of the relative motion of the atoms involved. This present work will discuss the nature of the Debye-
Waller factor in EXAFS spectra which contain a significant multiple scattering component.

\section{THE GENERAL FORMALISM}

The problem of thermal diffuse scattering is important in any structural tool which uses the difference in the phase of a scattered wave from different scattering sites to obtain structural information. In general, the effects of thermal vibrations are included by setting the distance between the scattering sites to their equilibrium values and multiplying each term representing the scattered intensity by a factor of the form $\exp (-M)$ where $M$ is the Debye-Waller factor for the scattering process. We present below a general formalism which may readily be applied to most scattering problems including the EXAFS effect.

Consider the following general scattering problem. Suppose we have an $N$-particle system with masses $m_{i}$ $(i=1, \ldots, N)$. Let $\mathbf{r}_{i}$ denote the position vector of the $i$ th atom in its equilibrium position with respect to some arbitrary origin. If $\mathbf{u}_{i}$ is the displacement vector of the $i$ th atom from its equilibrium position then the instantaneous position of this atom is given by

$$
\mathbf{r}=\mathbf{r}_{i}+\mathbf{u}_{i} \text {. }
$$

Let us consider now the scattering process whereby a particle incident in the direction $\mathbf{k}$ is scattered by atom $i$ into the direction $\mathbf{k}^{\prime}$. The probability amplitude $a_{i}$ for this event in the absence of thermal vibrations is

$$
a_{i}=\left\langle k^{\prime}\left|T_{i}\right| k\right\rangle=a_{i}^{0} e^{i\left(\mathbf{k}-\mathbf{k}^{\prime}\right) \cdot \mathbf{r}_{i}},
$$

where $T_{i}$ is the $T$ operator associated with the scattering potential at $\mathbf{r}=\mathbf{r}_{i} . a_{i}^{0}$ is the probability amplitude for the same scattering event but located at the origin. ${ }^{10}$ At finite temperatures, however, the position of the $i$ th atom is given by Eq. (1) so that the time dependent probability amplitude $A_{i}$ may be written as

$$
A_{i}=a_{i}^{0} e^{i K \cdot\left(\mathbf{r}_{i}+\mathbf{u}_{i}\right)}=a_{i} e^{i \mathbf{K} \cdot \mathbf{u}_{i}},
$$


where $\mathbf{K}=\mathbf{k}-\mathbf{k}^{\prime}$ and $\hbar \mathbf{K}$ represents the momentum transferred to the scattering atom. The total probability amplitude is then the sum of the amplitudes $A_{i}$ due to the individual scattering centers

$$
A=\sum_{i} A_{i}=\sum_{i} a_{i} e^{i \mathbf{K} \cdot \mathbf{u}_{i}}
$$

The total scattered intensity is then proportional to

$$
|A|^{2}=\sum_{i} \sum_{j} a_{i} a_{j}^{*} e^{\left.i \mathbf{K} \cdot \mathbf{u}_{i}-\mathbf{u}_{j}\right)}
$$

Throughout this present work the harmonic or quadratic approximation is assumed. While this approximation is strictly valid only for small displacement or amplitudes of vibration, it does however, provide a reasonably accurate description of most solids and molecules particularly at low temperatures. ${ }^{9}$ To study further the temperature dependence of the scattered intensity in the quadratic approximation it is convenient to introduce the normal coordinates $Q_{n}$ $(n=1, \ldots, 3 N)$. These coordinates have the advantage that they diagonalize both the potential and kinetic energy matrices of the system. The displacment vectors $\mathbf{u}_{i}$ may be expressed in this coordinate system as

$$
\mathbf{u}_{i} m_{i}^{1 / 2}=\sum_{n} \epsilon_{i}^{n} Q_{n}
$$

or

$$
\mathbf{u}_{i}=\sum_{n} \mathbf{e}_{i}^{n} Q_{n},
$$

where $\mathrm{e}_{i}^{n}=\epsilon_{i}^{n} / m_{i}^{1 / 2}$ and represents the amplitude vector of the $i$ th atom in the $n$th normal mode. The transformation between mass weighted and normal coordinates is orthogonal and therefore the amplitude vectors $\epsilon_{i}^{n}$ form an orthonormal set defined by ${ }^{11}$

$$
\sum_{i} \epsilon_{i}^{n} \cdot \epsilon_{i}^{n^{\prime}}=\delta_{n n^{\prime}}
$$

This property of normal coordinates eliminates the interaction or cross terms which occur in the potential and kinetic energies of the system. Equation (7) also serves to normalize the amplitudes of vibration of the atoms in each normal mode.

With the aid of Eq. (6) the expression for the scattered intensity [Eq. (5)] may be rewritten as

$$
|A|^{2}=\sum_{i} \sum_{j} a_{i} a_{j}^{*} \prod_{n} e^{i \mu_{i j}^{n} Q_{n}},
$$

where $\mu_{i j}^{n}=\mathbf{K} \cdot\left(\mathbf{e}_{i}^{n}-\mathbf{e}_{j}^{n}\right)$ and the sum in the exponent of Eq. (5) has been expressed as a product of exponential terms.

To obtain the experimentally observed intensity we must perform an ergodic average of Eq. (8) over a time period which is short on a microscopic scale but which is long compared with the period of vibration of the atoms involved. In practice, however, it is more convenient to perform an ensemble average of $\mathrm{Eq}$. (8) over a canonical ensemble defined by the Hamiltonian of the system. Classically this average is understood to be a mean over all possible initial states weighted with the corresponding Boltzmann factor. Assuming that the vibration of the atoms may be described by a quadratic Hamiltonian it may be shown that ${ }^{12}$

$$
\left\langle\exp \left(i \mu_{i j}^{n} Q_{n}\right)\right\rangle=\exp \left[-\frac{1}{2}\left(\mu_{i j}^{n}\right)^{2}\left\langle Q_{n}{ }^{2}\right\rangle\right],
$$

where the brackets $\langle. .$.$\rangle denote the ensemble or thermal$ average.

In the case of a harmonic oscillator the mean square amplitude of vibration $\left\langle Q_{n}^{2}\right\rangle$ may be related to the average energy of the oscillator $\left\langle E_{n}\right\rangle,{ }^{13}$

$\left\langle Q_{n}^{2}\right\rangle=\frac{\left\langle E_{n}\right\rangle}{\omega_{n}^{2}}=\frac{1}{\omega_{n}^{2}}\left[\frac{\hbar \omega_{n}}{\exp \left(\hbar \omega_{n} / k_{B} T\right)-1}+\frac{1}{2} \hbar \omega_{n}\right]$,

where $\omega_{n}$ is the frequency of the $n$th normal mode and $k_{B}$ is Boltzmann's constant. The expression for the thermally averaged scattered intensity may now be written as

$$
\left\langle|A|^{2}\right\rangle=\sum_{i} \sum_{j} a_{i} a_{j}^{*} e^{-M_{i j}},
$$

where $M_{i j}=\frac{1}{2} \sum_{n}\left(\mu_{i j}^{n}\right)^{2}\left\langle Q_{n}^{2}\right\rangle$ and the product of exponential terms in Eq. (8) has been replaced by a sum in the exponent of Eq. (11). The exponent $M_{i j}$ represents the Debye-Waller factor for the scattering process.

For computational purposes Eq. (10) may be conveniently written in terms of a hyperbolic cotangent ${ }^{14}$

$$
\left\langle Q_{n}^{2}\right\rangle=\frac{\hbar}{2 \omega_{n}} \operatorname{coth}\left(\frac{\hbar \omega_{n}}{2 k_{B} T}\right) .
$$

For low frequency modes or at high temperature the mean square amplitude of vibration may be approximated as

$$
\left\langle Q_{n}^{2}\right\rangle \approx k_{B} T / \omega_{n}^{2}
$$

and the Debye-Waller varies linearly with temperature. At low temperatures or for very high frequency modes the mean square amplitude of vibration may be written as

$$
\left\langle Q_{n}^{2}\right\rangle \approx \frac{1}{2} \hbar / \omega_{n}
$$

and the Debye-Waller factor is temperature independent.

\section{THE EXAFS PROBLEM}

While EXAFS is primarily a single scattering probe in certain instances low-order multiple scattering events contribute significantly to the extended structure. $\mathrm{Teo}^{7}$ and Boland et al. ${ }^{8}$ have shown that a three-atom system comprised of an absorbing atom and two neighboring atoms is sufficient to account for all significant multiple scattering effects in EXAFS. The phase and the amplitude of the multiple scattering components were shown to be a function of the geometry of the triatomic system. ${ }^{8}$ An EXAFS analysis based on a knowledge of these components will then allow bond angles to be determined.

To study the effect of thermal vibrations on these multiple scattering EXAFS components let us consider the threeatom system shown in Fig. 1. We shall first consider the general case in which the system has $C_{s}$ symmetry. The instantaneous positions of the atoms with respect to each other are also shown in Fig. 1. The expression for the EXAFS of this three-atom system in the absence of thermal vibrations is given by ${ }^{8}$

$$
\chi^{0}(k, \hat{e})=\chi_{s}^{0}(k, \hat{e})+2 \chi_{D}^{0}(k, \hat{e})+\chi_{T}^{0}(k, \hat{e}) .
$$




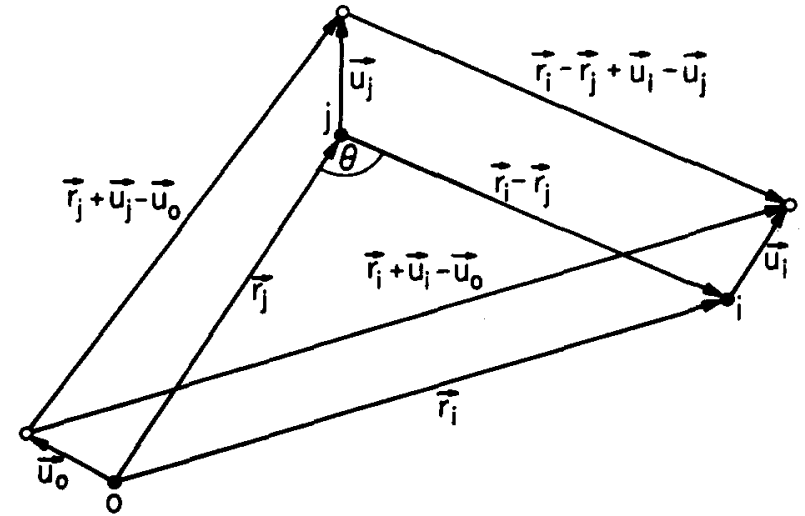

FIG. 1. The general three-atom system. The equilibrium positions of the central atom $(O)$, first nearest neighbor atom $(j)$ and second nearest neighbor atom $(i)$ are shown as filled circles. The displacement of these atoms from their respective equilibrium positions are shown as open circles. $\theta$ represents the equilibrium bridging angle. The equilibrium position of the central atom was chosen to represent the origin of the system.

The superscript zero indicates the absence of vibrations and $k$ is the photoelectron wave number defined by $k=\left[2 m\left(\hbar \omega-E_{0}\right)\right]^{1 / 2} / \hbar$, where $\omega$ is the photon frequency and $E_{0}$ is the threshold energy. The subscripts $S, D$, and $T$ refer to single, double, and triple scattering events, respectively, and $\hat{e}$ is the unit vector in the direction of polarization of the incident $\mathrm{x}$-ray beam.

The single scattering EXAFS, $\chi_{s}^{0}(k, \hat{e})$, consists of two terms each corresponding to single scattering events involving the neighboring atoms at $\mathbf{r}=\mathbf{r}_{i}$ and $\mathbf{r}=\mathbf{r}_{j}$ [see Figs. 2(a) and $2(b)] \cdot \chi_{s}^{0}(k, \hat{e})$ may be expressed as

$$
\chi_{s}^{0}(k, \hat{e})=\sum_{\alpha=i, j} \chi_{s_{\alpha}}^{0}(k, \hat{e}),
$$

where

$$
\begin{aligned}
\chi_{s_{\alpha}}^{0}(k, \hat{e})= & -\frac{3\left(\hat{r}_{\alpha} \cdot \hat{e}\right)^{2}}{k r_{\alpha}^{2}}\left(\frac{-m(2 \pi)^{2}}{\hbar^{2}}\right) \\
& \times \operatorname{Im}\left[\left\langle-k_{\alpha}\left|T_{\alpha}\right| k_{\alpha}\right\rangle e^{2 \mid \mathbf{k}_{\alpha} \cdot \boldsymbol{r}_{\alpha}} e^{2 i \delta_{1}}\right]
\end{aligned}
$$

and $\mathbf{k}=k \hat{r}_{\alpha}$. The $T$-matrix element represents the probability amplitude for scattering a photoelectron incident in di-

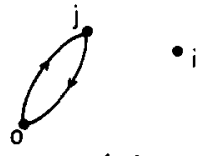

(a)

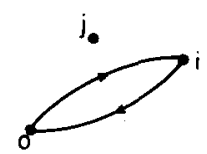

(b)

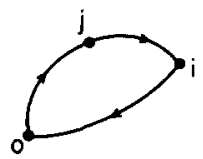

(c)

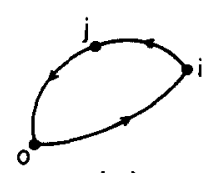

(d)

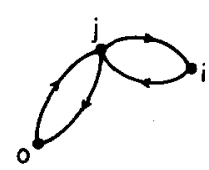

(e)
FIG. 2. The five significant scattering paths in a system of three atoms. (a) and (b) represent the single scattering paths from atoms $i$ and $j$. The two double scattering paths (c) and (d) are identical by virtue of time-reversal symmetry. The triple scattering path is shown in (e). rection $\mathbf{k}_{\alpha}$ into direction $-\mathbf{k}_{\alpha}$ and $\delta_{1}$ is the phase shift due to the central atom potential. The double scattering EXAFS, $\chi_{D}^{0}(k, \hat{e})$, involves events in which the photoelectron successively scatters off one neighboring atom and then scatters off the other remaining neighboring atom. The order in which the scattering occurs does not effect the probability amplitude so that this term is counted twice in Eq. (15) [see Figs. $2(c)$ and $2(d)] \cdot \chi_{D}^{0}(k, \hat{e})$ may be written as

$$
\begin{aligned}
\chi_{D}^{0}(k, \hat{e})= & \frac{-3\left(\hat{r}_{i} \cdot \hat{e}\right)\left(\hat{r}_{j} \cdot \hat{e}\right)}{k r_{i} r_{j} r_{i j}}\left(\frac{m^{2}(2 \pi)^{4}}{\hbar^{4}}\right) \\
& \times \operatorname{Im}\left\{\left(-k_{i}\left|T_{i}\right| k_{i j}\right\rangle\left\langle k_{i j}\left|T_{j}\right| k_{j}\right\rangle\right. \\
& \left.\times \exp \left[i\left(\mathbf{k}_{j} \cdot \mathbf{r}_{j}+\mathbf{k}_{i j} \cdot \mathbf{r}_{i j}+\mathbf{k}_{i} \cdot \mathbf{r}_{i}\right)\right] e^{2 i i_{1}}\right\},
\end{aligned}
$$

where $\mathbf{k}_{i j}=k \hat{r}_{i j}=k\left(\mathbf{r}_{i}-\mathbf{r}_{j}\right) /\left|\mathbf{r}_{i}-\mathbf{r}_{j}\right|$. The triple scattering EXAFS term $\chi_{T}^{0}(k, \hat{e})$ describes a process in which the outgoing photoelectron first scatters off the nearest neighboring atom $j$ then scatters off atom $i$ and finally scatters off atom $j$ once again [see Fig. $2(\mathrm{e})] \cdot \chi_{T}^{0}(k, \hat{e})$ may be written as

$$
\begin{aligned}
\chi_{T}^{0}(k, \hat{e})= & \frac{-3\left(\hat{r}_{j} \cdot \hat{e}\right)^{2}}{k r_{j}^{2} r_{i j}^{2}}\left(\frac{-m^{3}(2 \pi)^{6}}{\hbar^{6}}\right) \operatorname{Im}\left\{\left(-k_{j}\left|T_{j}\right|-k_{i j}\right)\right. \\
& \times\left\langle-k_{i j}\left|T_{i}\right| k_{i j}\right\rangle\left\langle k_{i j}\left|T_{j}\right| k_{j}\right\rangle \\
& \left.\times \exp \left[2 i\left(\mathbf{k}_{j} \cdot \mathbf{r}_{j}+\mathbf{k}_{i j} \cdot \mathbf{r}_{i j}\right)\right] e^{2 i \delta_{1}}\right\} .
\end{aligned}
$$

An expression for the EXAFS due to the instantaneous configuration of three-atom system shown in Fig. 1 may be obtained by replacing the relative equilibrium position vectors of the atoms by their respective instantaneous position vectors. Retaining only the lowest order terms in the displacement, the EXAFS, $\chi(k, e)$, due to this configuration is given by

$$
\begin{aligned}
\mathcal{\chi}(k, \hat{e})= & \chi_{s}^{0} \exp \left[2 i k \hat{r}_{\alpha} \cdot\left(\mathbf{u}_{\alpha}-\mathbf{u}_{0}\right)\right]+2 \chi_{D}^{0} \\
& \times \exp \left\{i k\left[\hat{r}_{j} \cdot\left(\mathbf{u}_{j}-\mathbf{u}_{0}\right)+\hat{r}_{i j} \cdot\left(\mathbf{u}_{i}-\mathbf{u}_{j}\right)\right]\right\} \\
& \times \exp \left\{i k\left[\hat{r}_{i} \cdot\left(\mathbf{u}_{i}-\mathbf{u}_{0}\right)\right]\right\}+\chi_{T}^{0} \\
& \times \exp \left\{2 i k\left[\hat{r}_{j} \cdot\left(\mathbf{u}_{j}-\mathbf{u}_{0}\right)+\hat{r}_{i j} \cdot\left(\mathbf{u}_{i}-\mathbf{u}_{j}\right)\right]\right\},
\end{aligned}
$$

where $\alpha=i, j$ and $\mathbf{u}_{i}, \mathrm{u}_{j}$, and $\mathrm{u}_{0}$ are the displacement vectors of neighboring atoms $i$ and $j$ and the central atom from their respective equilibrium positions. Note that we have neglected any changes that occur in the scattering angles due to the displacement of the atoms. Such angular changes are a higher order effect and may be treated separately. ${ }^{19}$ We may now proceed as described in the previous section by expressing the displacement vectors in terms of normal coordinates. Thus,

$$
\mathbf{u}_{i}=\sum_{n} \mathbf{e}_{i}^{n} Q_{n}
$$

and we may rewrite Eq. (19) as

$$
\begin{aligned}
\chi(k, \hat{e})= & \chi_{s}^{0} \exp \left(2 i k \sum_{n} \mu_{\infty 0}^{n} Q_{n}\right) \\
& +2 \chi_{D}^{0} \exp \left[i k \sum_{n}\left(\mu_{j}^{n}+\mu_{i j}^{n}+\mu_{j 0}^{n}\right) Q_{n}\right] \\
& +\chi_{T}^{0} \exp \left[2 i k \sum_{n}\left(\mu_{j 0}^{n}+\mu_{i j}^{n}\right) Q_{n}\right],
\end{aligned}
$$

where $\mu_{i j}^{n}=\hat{r}_{i j} \cdot\left(\mathbf{e}_{i}^{n}-\mathbf{e}_{j}^{n}\right)$ and represents the maximum effective change in the distance along the equilibrium internuclear axis between atoms $i$ and $j$ in the $n$th normal mode of 
vibration. A similar interpretation applies to the terms $\mu_{j 0}^{n}$ and $\mu_{i o}^{n}$. For the three-atom system under consideration there are in total nine degrees of freedom so that the sum over $n$ above extends to three terms for nonlinear systems and four terms for linear systems.

The experimentally observed EXAFS may be obtained by performing an ensemble average of Eq. (21) as described in Sec. II. Using Eq. (9) above, the thermally averaged EXAFS for the general system of $C_{s}$ symmetry is then given by $\langle\chi(k, \hat{e})\rangle=\chi_{s}^{0} \exp \left[-2 k^{2} \sum_{n}\left(\mu_{\alpha 0}^{n}\right)^{2}\left\langle Q_{n}^{2}\right\rangle\right]$

$$
\begin{aligned}
& +2 \chi_{D}^{0} \exp \left[-\frac{1}{2} k^{2} \sum_{n}\left(\mu_{j 0}^{n}+\mu_{i j}^{n}+\mu_{i 0}^{n}\right)^{2}\left\langle Q_{n}^{2}\right\rangle\right] \\
& +\chi_{T}^{0} \exp \left[-2 k^{2} \sum_{n}\left(\mu_{j 0}^{n}+\mu_{i j}^{n}\right)^{2}\left\langle Q_{n}^{2}\right\rangle\right] .
\end{aligned}
$$

The exponential terms in Eq. (22) correspond to the DebyeWaller factors for each of the scattering processes. The Debye-Waller factor obtained for the single scattering EXAFS is identical to that found by previous investigators. ${ }^{3,4}$

\section{APPLICATION TO MODEL SYSTEMS}

For our present purpose it is convenient to write out Eq. (22) in detail:

$$
\begin{aligned}
\langle\chi(k, \hat{e})\rangle= & \chi_{s}^{0} \exp \left\{-2 k^{2} \sum_{n}\left[\left(\hat{r}_{\alpha} \cdot \mathbf{e}_{\alpha}^{n}\right)-\left(\hat{r}_{\alpha} \cdot \mathbf{e}_{0}^{n}\right)\right]^{2}\left\langle Q_{n}^{2}\right\rangle\right\} \\
& +2 \chi_{D}^{0} \exp \left\{-\frac{1}{2} k^{2} \sum_{n}\left[\left(\hat{r}_{j} \cdot \mathbf{e}_{j}^{n}\right)-\left(\hat{r}_{j} \cdot \mathbf{e}_{0}^{n}\right)+\left(\hat{r}_{i j} \cdot \mathbf{e}_{i}^{n}\right)-\left(\hat{r}_{i j} \cdot \mathbf{e}_{j}^{n}\right)+\left(\hat{r}_{i} \cdot \mathbf{e}_{i}^{n}\right)-\left(\hat{r}_{i} \cdot \mathbf{e}_{o}^{n}\right)\right]^{2}\left\langle Q_{n}^{2}\right\rangle\right\} \\
& \left.+\chi_{T}^{0} \exp \left\{-2 k^{2} \sum_{n}\left[\hat{r}_{j} \cdot \mathbf{e}_{j}^{n}\right)-\left(\hat{r}_{j} \cdot \mathbf{e}_{0}^{n}\right)+\left(\hat{r}_{i j} \cdot \mathbf{e}_{i}^{n}\right)-\left(\hat{r}_{i j} \cdot \mathbf{e}_{j}^{n}\right)\right]^{2}\left\langle Q_{n}^{2}\right\rangle\right\},
\end{aligned}
$$

where $\alpha=i, j$. Now consider that the three atom is symmetric and bent with a bridging angle $\theta$ and belongs to the $C_{2 v}$ point group. The normal modes of vibration transform as

$$
\Gamma_{\text {vib }}=2 A_{1}+B_{1} \text {. }
$$

The two totally symmetric $A_{1}$ modes correspond to a symmetric stretching and bending mode of vibration. The $B_{1}$ mode is associated with the asymmetric stretch. A schematic representation of the normal modes of the three-atom system are shown in Fig. 3. Note that all displacement vectors are confined to the plane defined by the three atoms.

The symmetry of the system results in a simplification of Eq. (23) which is dependent on the normal mode in question. All normal modes in the $C_{2 v}$ point group are subject to the following conditions:

For all modes $\quad m_{0}=m_{i}=m$,

in a $C_{2 v}$ point group: $\quad\left|\mathbf{e}_{0}^{n}\right|=\left|\mathbf{e}_{i}^{n}\right|$,

where $m_{0}$ and $m_{i}$ are the masses of the central atom and neighboring atom $i$, respectively. In addition, a study of either Figs. $3(\mathrm{a})$ or 3 (b) shows that the following restrictions apply to both $A_{1}$ type modes of vibration:

$$
\begin{aligned}
& \left(\hat{r}_{j} \cdot \mathbf{e}_{0}^{A_{1}}\right)=-\left(\hat{r}_{i j} \cdot \mathbf{e}_{i}^{A_{1}}\right), \\
& A_{1} \text { mode: } \\
& \left(\hat{r}_{j} \cdot \mathbf{e}_{j}^{A_{1}}\right)=-\left(\hat{r}_{i j} \cdot \mathbf{e}_{j}^{A_{1}}\right), \\
& \left(\hat{r}_{i} \cdot \mathbf{e}_{0}^{A_{1}}\right)=-\left(\hat{r}_{i} \cdot \mathbf{e}_{i}^{A_{1}}\right) .
\end{aligned}
$$

An analogous set of conditions apply to the $B_{1}$ mode of vibration [see Fig. 3(c)]

$$
\begin{aligned}
& \left(\hat{r}_{j} \cdot \mathbf{e}_{0}^{B_{1}}\right)=\left(\hat{r}_{i j} \cdot \mathbf{e}_{i}^{B_{1}}\right), \\
B_{1} \text { mode: } & \left(\hat{r}_{i j} \cdot \mathbf{e}_{j}^{B_{1}}\right)=\left(\hat{r}_{j} \cdot \mathbf{e}_{j}^{B_{1}}\right), \\
& \left(\hat{r}_{i} \cdot \mathbf{e}_{0}^{B_{1}}\right)=\left(\hat{r}_{i} \cdot \mathbf{e}_{i}^{B_{1}}\right) .
\end{aligned}
$$

With the aid of Eqs. (25), (26), and (27) the thermally averaged EXAFS, Eq. (23), for a three-atom system of $C_{2 v}$ symmetry may be reduced to

$$
\begin{aligned}
\langle\chi(k, \hat{e})\rangle_{C_{2 v}=}= & \chi_{s_{j}}^{0} \exp \left\{-2 k^{2} \sum_{A_{1}}\left[\left(\hat{r}_{j} \cdot \mathbf{e}_{j}^{A_{1}}\right)-\left(\hat{r}_{j} \cdot \mathbf{e}_{0}^{A_{1}}\right)\right]^{2}\left\langle Q_{A_{1}}^{2}\right\rangle\right\} \exp \left\{-2 k^{2}\left[\left(\hat{r}_{j} \cdot \mathbf{e}_{j}^{B_{1}}\right)-\left(\hat{r}_{j} \cdot \mathbf{e}_{0}^{B_{1}}\right)\right]^{2}\left\langle Q_{B_{1}}^{2}\right\rangle\right\} \\
& +\chi_{s_{i}}^{0} \exp \left\{-2 k^{2} \sum_{A_{1}}\left[2\left(\hat{r}_{i} \cdot \mathbf{e}_{i}^{A_{1}}\right)\right]^{2}\left\langle Q_{A_{1}}^{2}\right\rangle\right\}+2 \chi_{D}^{0} \exp \left\{-\frac{1}{2} k^{2} \sum_{A_{1}}\left[2\left(\hat{r}_{i j} \cdot \mathbf{e}_{i}^{A_{1}}\right)+2\left(\hat{r}_{j} \cdot \mathbf{e}_{j}^{A_{1}}\right)+2\left(\hat{r}_{i} \cdot \mathbf{e}_{i}^{A_{1}}\right)\right]^{2}\left\langle Q_{A_{1}}^{2}\right)\right\} \\
& +\chi_{T}^{0} \exp \left\{-2 k^{2} \sum_{A_{1}}\left[2\left(\hat{r}_{i j} \cdot \mathbf{e}_{i}^{A_{1}}\right)+2\left(\hat{r}_{j} \cdot \mathbf{e}_{j}^{A_{i}}\right)\right]^{2}\left\langle Q_{A_{1}}^{2}\right\rangle\right\}
\end{aligned}
$$


where $\chi_{s_{i}}^{0}$ and $\chi_{s_{f}}^{0}$ are the single scattering contributions to the EXAFS from atoms $i$ and $j$ in the absence of thermal vibrations. The Debye-Waller factors in Eq. (28) have been factored into two terms to emphasize the separate contributions of the $A_{1}$ and $B_{1}$ type modes. Note that the double and triple EXAFS components receive no contribution to their Debye-Waller factors from the $B_{1}$ normal mode. The asymmetric $B_{1}$ mode contributions only to the single scattering Debye-Waller factor for the first shell atom.

A calculation of the Debye-Waller factors for the $C_{2 v}$ system requires a full normal mode analysis. Before doing so it is interesting to consider a linear symmeric configuration as a limiting case of a system with $C_{2 v}$ symmetry. Suppose that the interatomic distances $r_{j}$ and $r_{i j}$ are equal and the terminal atoms are of the same kind. Then the system belongs to the $D_{\infty} h$ point group and the normal modes of vibration transform as

$$
\Gamma_{\text {vib }}=\Sigma_{g}^{+}+\Sigma_{u}^{+}+\Pi_{u} \text {. }
$$

A schematic representation of the normal modes of the system are shown in Fig. 4. The bending modes $\Pi_{u}$ are degenerate and may be interconverted by a rotation of $90^{\circ}$ about the axis of the molecule.

The symmetry of the system requires that the following condition must always be satisfied:

$$
\begin{array}{ll} 
& m_{0}=m_{i}=m . \\
\text { For all modes: } & \hat{r}_{j}=\hat{r}_{i}=\hat{r}_{i j}, \\
\text { in a } D_{\infty h} \text { group } & \left|\mathbf{e}_{n}^{o}\right|=\left|\mathbf{e}_{i}^{n}\right| .
\end{array}
$$

In addition, the following restrictions apply to each of the normal modes of vibration (see Fig. 4):

$$
\begin{aligned}
& \Sigma_{g}^{+} \text {mode: }\left(\hat{r}_{j} \cdot \mathbf{e}_{0}^{\Sigma_{g}^{+}}\right)=-\left(\hat{r}_{i j} \cdot \mathbf{e}_{i}^{\Sigma_{g}^{+}}\right), \quad\left|\mathbf{e}_{j}^{\Sigma_{g}^{+}}\right|=0 . \\
& \Pi_{u} \text { mode: }\left(\hat{r}_{j} \cdot \mathbf{e}_{0}^{\Pi_{u}}\right)=\left(\hat{r}_{j} \cdot \mathbf{e}_{i}^{I_{u}}\right)=\left(\hat{r}_{j} \cdot \mathbf{e}_{j}^{I_{u}}\right)=0 . \\
& \Sigma_{u}^{+} \operatorname{mode}:\left(\hat{r}_{j} \cdot \mathbf{e}_{0}^{\Sigma_{g}^{+}}\right)=\left(\hat{r}_{j} \cdot \mathbf{e}_{i}^{\Sigma_{u}^{+}}\right) .
\end{aligned}
$$

With the above conditions satisfied, the expression for the thermally averaged EXAFS in a three-atom system of symmetry $D_{\infty h}$ is given by

$$
\begin{aligned}
\langle\chi(k, \hat{e})\rangle_{D_{\infty h}}= & \chi_{s_{j}}^{0} \exp \left\{-2 k^{2}\left(\hat{r}_{j} \cdot \mathbf{e}_{0}^{\Sigma_{g}^{+}}\right)^{2}\left\langle Q_{\Sigma_{g}^{+}}^{2}\right\rangle\right\} \exp \left\{-2 k^{2}\left[\left(\hat{r}_{j} \cdot \mathbf{e}_{j}^{\Sigma_{u}^{+}}\right)-\left(\hat{r}_{j} \cdot \mathbf{e}_{0}^{\Sigma^{+}}\right)\right]^{2}\left\langle Q_{\Sigma_{u}^{+}}^{2}\right)\right\} \\
& +\chi_{s_{i}}^{0} \exp \left\{-2 k^{2}\left[2\left(\hat{r}_{i} \cdot \mathbf{e}_{i}^{\Sigma_{g}^{+}}\right)\right]^{2}\left\langle Q_{\Sigma_{g}^{+}}^{2}\right\rangle\right\}+2 \chi_{D}^{0} \exp \left\{-\frac{1}{2} k^{2}\left[4\left(\hat{r}_{i} \cdot \mathbf{e}_{i}^{\Sigma_{g}^{+}}\right)\right]^{2}\left\langle Q_{\Sigma_{g}^{+}}^{2}\right\rangle\right\} \\
& +\chi_{T}^{0} \exp \left\{-2 k^{2}\left[2\left(\hat{r}_{i} \cdot \mathbf{e}_{i}^{\Sigma_{g}^{+}}\right)\right]^{2}\left\langle Q_{\Sigma_{g}^{+}}^{2}\right\rangle\right]
\end{aligned}
$$

The degenerate bending modes of vibration do not contribute to any of the Debye-Waller factors in Eq. (32). This occurs because the displacement vectors in these modes have no components along the axis of the three-atom system. Furthermore, the asymmetric stretching mode $\Sigma_{u}^{+}$contributes only to the Debye-Waller factor in the first shell single scattering EXAFS.

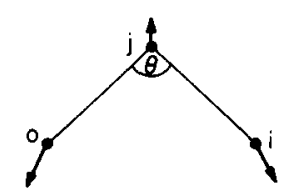

(a) A, STRETCH

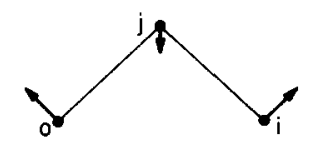

(b) $A_{1}$ BEND

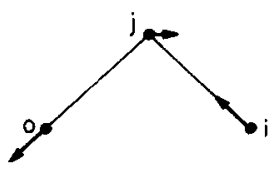

(c) B, STRETCH

FIG. 3. Schematic of the normal modes in a three-atom system of $C_{2 v}$ symmetry. There are three normal modes, two of $A_{1}$ type symmetry and a single $B_{1}$ mode.
To analyze the normal modes of the $C_{2 v}$ three-atom system it is convenient to introduce the internal displacement coordinates $R_{1}$ and $R_{2}$ together with an angle bending coordinate $\delta_{3} . R_{1}$ and $R_{2}$ represent the change in the bond
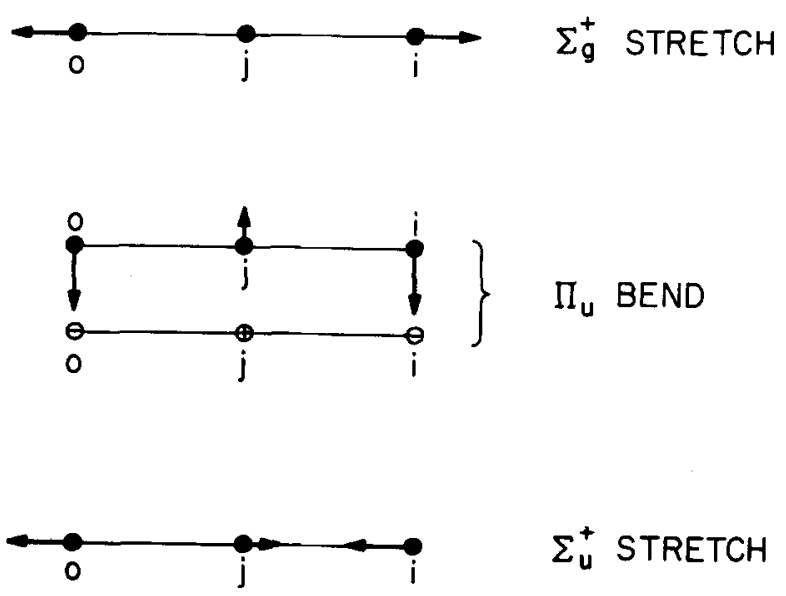

FIG. 4. Schematic of the normal modes in a three-atom system of $D_{\infty} h$ symmetry. The symmetric stretch $\Sigma_{g}{ }^{+}$involves no motion of the intervening atom $j$. The degenerate bonding modes $\Pi_{u}$ may be interconverted by a rotation of $90^{\circ}$ about the molecular axis. The asymmetric stretch $\Sigma_{u}^{+}$is the limiting case of the single $B_{1}$ mode in a $C_{2 v}$ system [Fig. 3(c)]. 


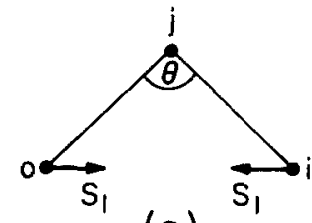

(a)

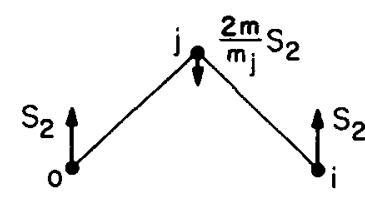

(b)

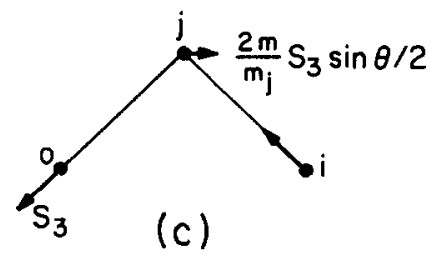

FIG. 5. The symmetrized coordinates used in a normal mode analysis of the three-atom system. $S_{1}$ and $S_{2}$ transform as $A_{1}$ in a $C_{2 v}$ system while $S_{3}$ transforms as $B_{1}$ and is identical, apart from a constant factor, to the asymmetric stretching mode.

lengths $r_{j}$ and $r_{i j}$ from their respective equilibrium values. The bending coordinate $\delta_{3}$ represents the change in the bridging angle $\theta$. For small amplitudes of vibration both the kinetic and potential energies of the system may be expressed as quadratic functions of these coordinates. In the instance of a $C_{2 v}$ three-atom system the potential energy may be written as

$$
2 V=a_{11}\left(R_{1}+R_{2}\right)+a_{33} \delta_{3}+a_{12} R_{1} R_{2},
$$

when the force constant $a_{11}=a_{22}$ and there is no interaction force constant between the displacement and bending coordinates.

For computational purposes it is more convenient to introduce the symmetry coordinates $S_{1}, S_{2}$, and $S_{3}$ shown in Fig. 5. Note that $S_{1}$ and $S_{2}$ transform as $A_{1}$ in the $C_{2 v}$ point group while $S_{3}$ transforms as $B_{1}$. The potential and kinetic energies may be written as

$$
2 V=C_{11} S_{1}^{2}+C_{22} S_{2}^{2}+C_{33} S_{3}^{2}+2 C_{12} S_{1} S_{2}
$$

and

$$
2 T=d_{11} \dot{S}_{1}^{2}+d_{22} \dot{S}_{2}^{2}+d_{33} \dot{S}_{3}^{2}+2 d_{12} \dot{S}_{1} \dot{S}_{2} .
$$

The two sets of force constants in Eqs. (33) and (34) are related to each other by ${ }^{15}$

$$
\begin{aligned}
& C_{11}=2 \sin ^{2}(\theta / 2)\left[a_{11}+a_{12}\right]+4 \cos ^{2}(\theta / 2) a_{33} / r_{j}^{2}, \\
& C_{12}=\left[2 a_{11} p-4 p a_{33} / r_{j}^{2}+2 a_{12} p\right] \sin (\theta / 2) \cos (\theta / 2), \\
& C_{22}=2 p^{2} \cos ^{2}(\theta / 2)\left[a_{11}+a_{12}\right]+4 p^{2} \sin ^{2}(\theta / 2) a_{33} / r_{j}^{2}, \\
& C_{33}=2 r^{2}\left(a_{11}-a_{12}\right)
\end{aligned}
$$

where $p=1+2 m / m_{j}$ and $r=1+\left(2 m / m_{j}\right) \sin ^{2}(\theta / 2)$. The coefficients in the expansion of the kinetic energy [Eq. (35)] may also be evaluated:

$$
\begin{aligned}
& d_{11}=2 m, \quad d_{22}=2 m p, \quad d_{33}=2 m r, \\
& d_{12}=d_{13}=d_{23}=0 .
\end{aligned}
$$

The frequency of each normal mode of vibration may now be expressed in terms of the force constants $a_{i j}$ and the bridging angle $\theta$ :

$\lambda_{3}=4 \pi^{2} c^{2} v_{3}^{2}=\left[\frac{1}{m}+\frac{2}{m_{j}} \sin ^{2}(\theta / 2)\right]\left(a_{11}-a_{12}\right)$,

$$
\begin{aligned}
& \lambda_{1}+\lambda_{2}= 4 \pi^{2} c^{2}\left(v_{1}^{2}+v_{2}^{2}\right)=\left[\frac{1}{m}+\frac{2}{m_{j}} \cos ^{2}(\theta / 2)\right] \\
& \times\left(a_{11}+a_{12}\right)+2\left[\frac{1}{m}+\frac{2}{m_{j}} \sin ^{2}(\theta / 2)\right] \frac{a_{33}}{r_{j}^{2}}, \\
& \lambda_{1} \lambda_{2}=16 \pi \pi^{4} c^{4} v_{1}^{2} v_{2}^{2}=2\left(\frac{1}{m^{2}}+\frac{2}{m m_{j}}\right)\left(a_{11}+a_{12}\right) \frac{a_{33}}{r_{j}^{2}}
\end{aligned}
$$

$\lambda_{1}$ and $\lambda_{2}$ are associated with the $A_{1}$ stretching and bending modes of vibration while $\lambda_{3}$ is associated with the asymmetric $B_{1}$ stretching mode. The normal modes of vibration may then be calculated from the secular equation defined by Eqs. (34) and (35):

$$
\begin{aligned}
Q_{A_{1}}\left(\lambda_{1}\right)= & \left(C_{22}-\lambda_{1} d_{22}\right) S_{1}-\left(C_{21}-\lambda_{1} d_{21}\right) S_{2}, \\
Q_{A_{1}}\left(\lambda_{2}\right)= & \left(C_{22}-\lambda_{2} d_{22}\right) S_{1}-\left(C_{21}-\lambda_{2} d_{21}\right) S_{2}, \\
Q_{B_{1}}\left(\lambda_{3}\right)= & {\left[\left(C_{11}-\lambda_{3} d_{11}\right)\left(C_{22}-\lambda_{3} d_{22}\right)\right.} \\
& \left.-\left(C_{21}-\lambda_{3} d_{21}\right)\left(C_{12}-\lambda_{3} d_{12}\right)\right] S_{3} .
\end{aligned}
$$

For the linear symmetric system of point group $D_{\infty} h$ the symmetry coordinates are identical to the normal coordinates shown in Fig. 4. The explicit form of the symmetrized force constants and the frequencies of the normal modes may be obtained by substituting a bridging angle of $180^{\circ}$ into Eqs. (36) and (38), respectively.

The approach we shall adopt in this present work is as follows. Given the normal modes of vibration for a linear symmetrical system we can calculate all the force constants from Eq. (38) when $\theta=180^{\circ}$ is substituted for the bridging angle. These force constants are then used to calculate the frequencies of the normal modes and the symmetrized force constants in Eqs. (38) and (36) for a system of $C_{2 v}$ symmetry with a bridging angle $\theta$. It is implicitly assumed that the force constants are independent of $\theta$ for the angular range of these calculations, $\theta=100^{\circ}-180^{\circ}$. This approximation is least valid for a description of the bending modes. Given this information, the explict form of the normal modes may be calculated from the secular equation in Eq. (39). The calculated frequency of each normal mode allows the mean square amplitude of vibration to be determined [Eq. (12)]. The contribution to the Debye-Waller factors from each normal mode may then be summed to obtain the total factor in Eq. (28) for each scattering path.

The systems chosen for this study are shown in Table $I$. The Debye-Waller factor at $10 \mathrm{~K}$ for each of these threeatom systems was calculated using Eq. (28) together with the normal modes calculated as described above. The results of these calculations are shown in Fig. 6. The calculated frequency of each of the normal modes of the $\mathrm{BeBr}_{2}$ compound as a function of bridging angle are shown in Fig. 7. This

TABLE I. Normal frequencies of the model systems."

\begin{tabular}{lcccc}
\hline \hline System & $v_{1}\left(\mathrm{~cm}^{-1}\right)$ & $v_{2}\left(\mathrm{~cm}^{-1}\right)$ & $v_{3}\left(\mathrm{~cm}^{-1}\right)$ & $m / m_{j}$ \\
\hline $\mathrm{AuBr}_{2}$ & 209 & 77 & 254 & 0.40 \\
$\mathrm{CuBr}_{2}$ & 193 & 81 & 322 & 1.26 \\
$\mathrm{CSe}_{2}$ & 364 & 313 & 1303 & 6.58 \\
$\mathrm{BeBr}_{2}$ & 230 & 220 & 1010 & 8.88 \\
$\mathrm{Bel}_{2}$ & 160 & 175 & 873 & 14.11 \\
\hline \hline
\end{tabular}

Reference 16 . 

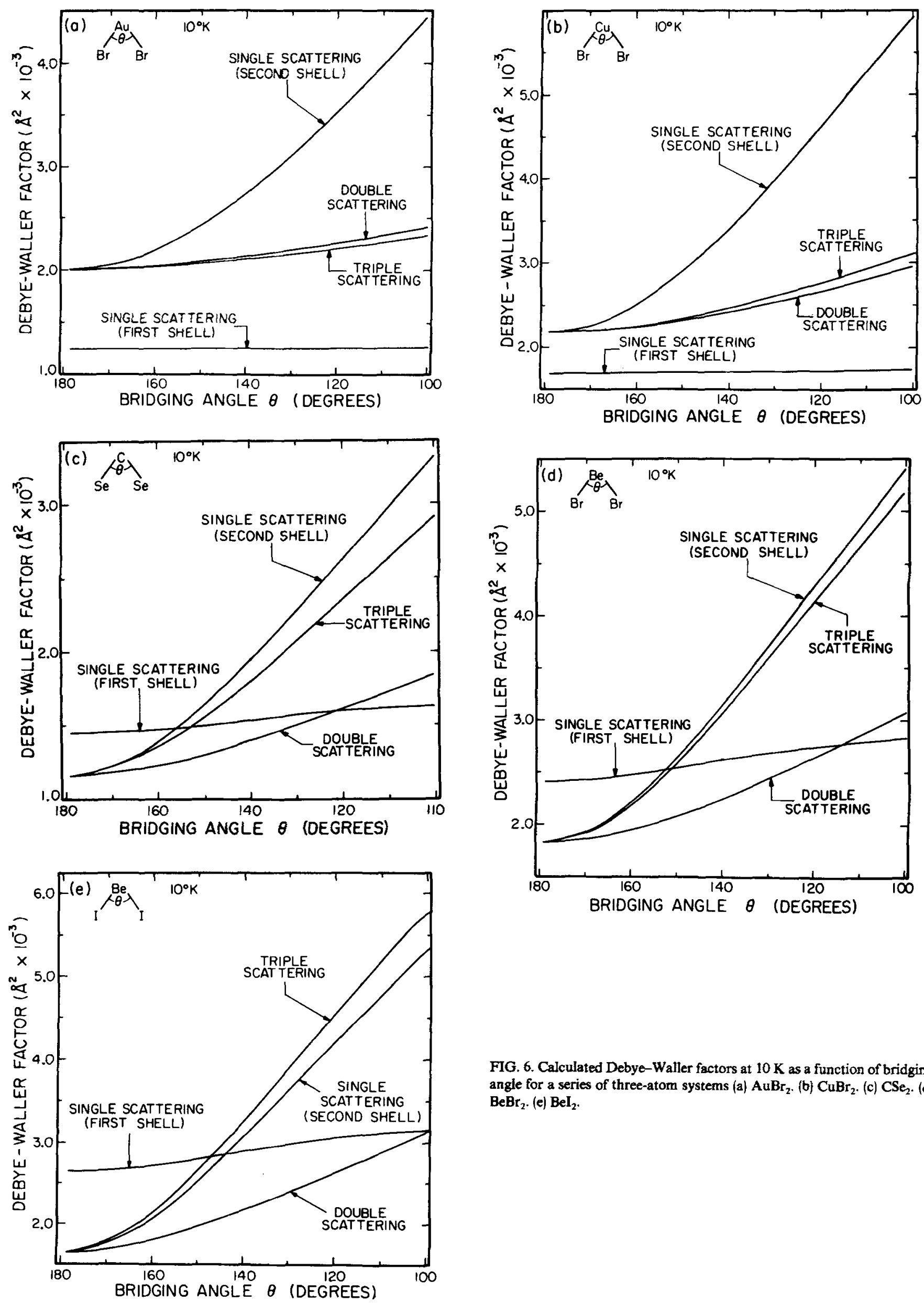

FIG. 6. Calculated Debye-Waller factors at $10 \mathrm{~K}$ as a function of bridging angle for a series of three-atom systems (a) $\mathrm{AuBr}_{2}$, (b) $\mathrm{CuBr}_{2}$. (c) $\mathrm{CSe}_{2}$. (d) $\mathrm{BeBr}_{2}$. (e) $\mathrm{BeI}_{2}$. 


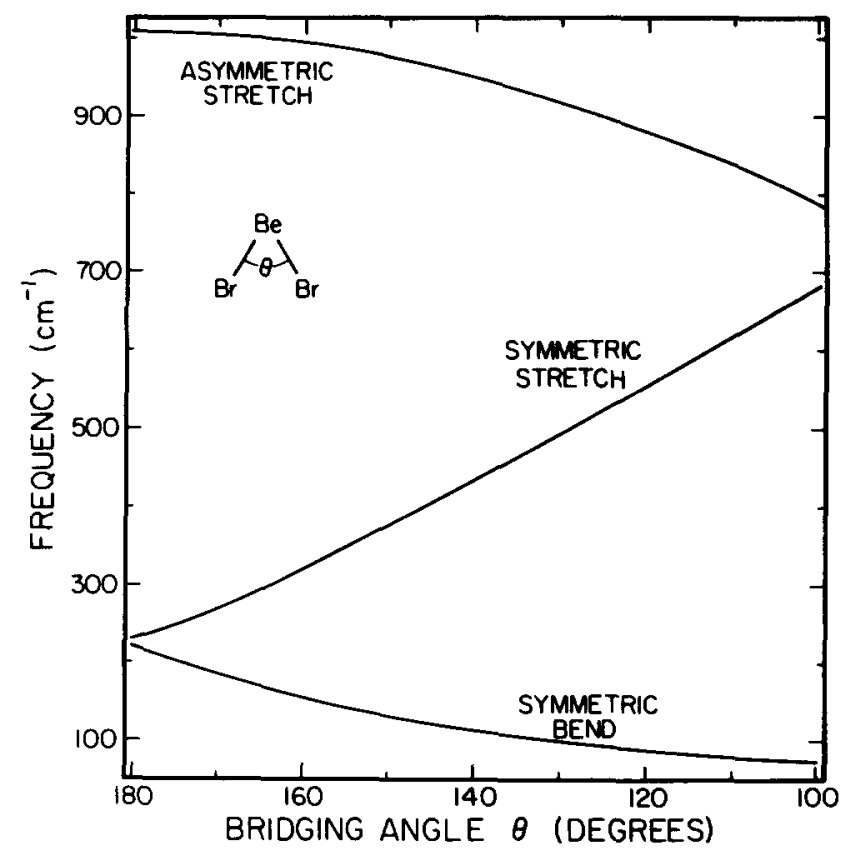

FIG. 7. Calculated frequencies of the normal modes of vibration for the $\mathrm{BeBr}_{2}$ system as a function of bridging angle $\theta$.

compound was chosen for a detailed study as is mass ratio is typical of that encountered in instances where multiple scattering is significant. The temperature dependence of the Debye-Waller factor for the $\mathrm{BeBr}_{2}$ system with three different bridging angles is shown in Fig. 8.

\section{DISCUSSION}

The Debye-Waller factors for the series of three-atom systems described in Table I are shown in Fig. 6. Note that the double scattering factor has been divided by four so that the Debye-Waller factor for each scattering path has the standard form exp $\left(-2 k^{2} \sigma^{2}\right)$ [see Eq. (28)]. At all temperatures the Debye-Waller factors associated with the second shell single scattering, the double scattering and the triple scattering EXAFS components converge to a single value at a bridging angle of $180^{\circ}$. This convergence is required by Eq. (32) which describes the Debye-Waller factor for a linear system as a limiting case of that for a similar but bent system.

The relative magnitudes of the Debye-Waller factors associated with the various scattering paths deserves comment. A normal mode analysis of each system shown in Table $I$ indicates that for all mass ratios $\left(m / m_{j}\right)$ at a bridging angle of $100^{\circ}$ the displacement vectors of the terminal atoms in the $A_{1}$ stretch make an angle of about $40^{\circ}$ with the base of the isosceles triangle which represents the system. As the system approaches linearity this angle decreases until it is zero when the bridging angle is $180^{\circ}$. In the case of the $A_{1}$ bend this angle depends on the mass ratio of the system. For small mass ratios the displacement vectors of the terminal atoms make an angle of about $150^{\circ}$ with the base of the triangle. When the mass ratio is larger this initial angle is closer to $180^{\circ}$. In both cases as the system becomes less bent this angle decreases until it is $90^{\circ}$ when a bridging angle of $180^{\circ}$ is achieved. The contribution of a given mode to the Debye-
Waller factor for a particular scattering path is dependent on the frequency of the mode due to the presence of the Boltzmann factor in Eq. (10). In general, both the asymmetric stretch and symmetric bend increase in frequency as the system becomes less bent while the frequency of the symmetric stretch decreases (see Fig. 7). Furthermore, different scattering paths show different degrees of sensitivity to the displacements of the atoms that occur in a given normal mode. For instance, the triple scattering path receives a large contribution to its Debye-Waller factor from the $A_{1}$ stretching mode. This scattering path is, however, very insensitive to the $A_{1}$ bending mode except perhaps at small bridging angles $\left(100^{\circ}\right)$ where the frequency of the bend is low and the displacement vectors of the terminal atoms still have significant components along the internuclear axes. At large mass ratios the contribution to the triple scattering Debye-Waller factor from the $A_{1}$ stretch increases as the frequencies of the two $A_{1}$ modes become comparable (see Table I). On the other hand, the second shell single scattering Debye-Waller factor receives a large contribution from the $A_{1}$ bend. This is particularly true at small bridging angles (where the frequency is low) and at high mass ratios where the displacement vectors of the terminal atoms have large components along the base of the isosceles triangle.

From Fig. 6 it is apparent that the first shell single scattering and the triple scattering Debye-Waller factors increase as the mass ratio increases. This occurs because both of these scattering paths are sensitive to the $A_{1}$ stretch whose frequency drops relative to the $A_{1}$ bend as the mass ratio increases. When the frequency of the $A_{1}$ bend is greater than the $A_{1}$ stretch the magnitude of the triple scattering DebyeWaller factor surpasses that of the second shell single scattering [see Fig. 6(e)]. The double scattering factor, however, is sensitive to both $A_{1}$ type modes. At small angles this scattering path receives a large contribution to the DebyeWaller factor from the $A_{1}$ bend. As the system approaches linearity the frequency of the $A_{1}$ bend increases and the components of the displacement vectors of the terminal atoms diminish so that the contribution from this mode decreases. The contribution to the Debye-Waller factor from the $A_{1}$ stretch increases, however, due to the reduced frequency of this mode at large angles.

The temperature dependence of the Debye-Waller factors for the $\mathrm{BeBr}_{2}$ system is shown in Fig. 8. The low frequency of the bending mode at small bridging angles $\left(100^{\circ}\right)$ causes the second shell single scattering Debye-Waller factor to have the observed sharp temperature dependence. The interplay of both $A_{1}$ modes in determining the Debye-Waller factor for the double scattering path is evident from Fig. 8. At small bridging angles the temperature dependence of the double scattering term is more severe than that for the triple scattering term. This occurs because the double scattering path is sensitive to the $A_{1}$ bend which has a low frequency at these angles while the triple scattering path is most sensitive to the $A_{1}$ stretch which occurs at much higher frequencies (see Fig. 7). As the system approaches linearity, however, the temperature dependence of both the double and triple scattering paths become very similar due to the dominant contribution of the $A_{1}$ stretch to each Debye-Waller factor. In 

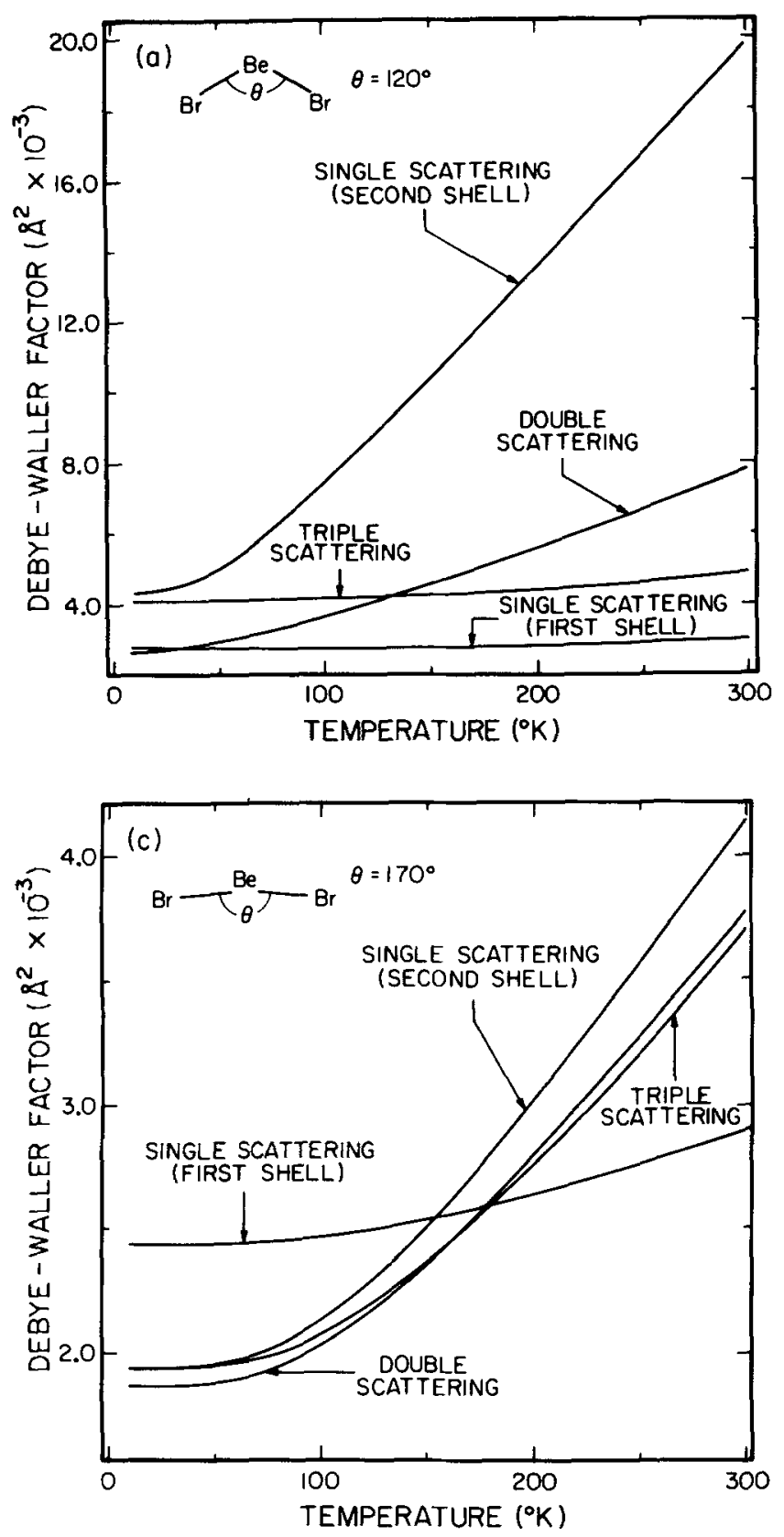

general, it is apparent that the Debye-Waller factors for each scattering path are strong functions of temperature. At sufficiently low temperatures each Debye-Waller factor is independent of temperature while at higher temperature the Debye-Waller factors vary linearly with temperature in agreement with Eqs. (13) and (14).

The purpose of this present study is to provide an understanding of the Debye-Waller factor in EXAFS so that accurate bond angles may be determined. A standard method of analysis for EXAFS data that contain a significant multiple scattering component has not yet been presented. Teo' has suggested that the terms involving the second shell atom in Eq. (15) may be combined and that the resulting expression can be analyzed as though it described a single scattering EXAFS process with a modified amplitude and phase. This results in a considerable simplification of the data analysis. This approach, however, neglects the polarization terms in Eq. (17). In addition, it assumes that the

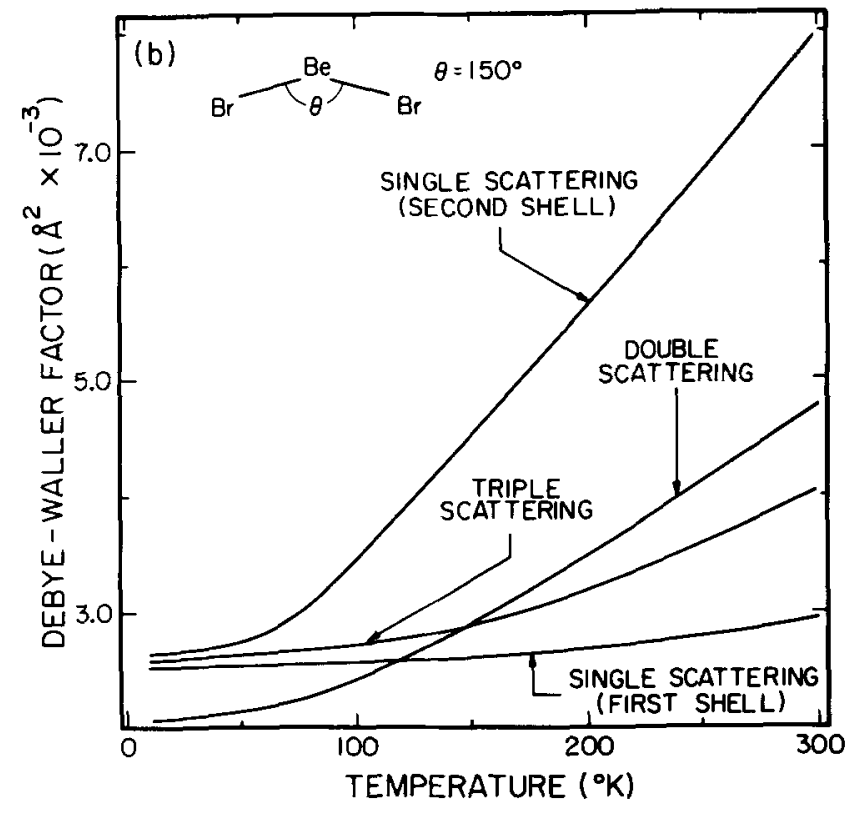

FIG. 8. Temperature dependence of the Debye-Waller factors for the $\mathrm{BeBr}_{2}$ system at three bridging angles. (a) $\theta=120^{\circ}$. (b) $\theta=150^{\circ}$. (c) $\theta=170^{\circ}$.

Debye-Waller factors for all scattering paths involving the second shell atom are the same. To study the significance of this latter assumption the resulting modification in the amplitude of the double and triple EXAFS components in the $\mathrm{BeBr}_{2}$ system were calculated and are shown in Fig. 9. All amplitude calculations were carried out at $k=10 \AA^{-1}$, the amplitude modification is smaller and larger at smaller and larger values of $k$, respectively. The errors involved are clearly larger at small bridging angles and high temperatures. At low temperatures, the larger modification occurs in the amplitude of the double scattering component while at higher temperatures the amplitude of the triple scattering component is more sensitive. For a bridging angle of $120^{\circ}$ the amplitudes of the double and triple scattering terms are reduced by a factor of 11.1 and 19.4, respectively, at room temperature. The reductions in the amplitudes are considerably less for a system that is approximately linear, especially at low temperature [see Fig. 9(d)]. 

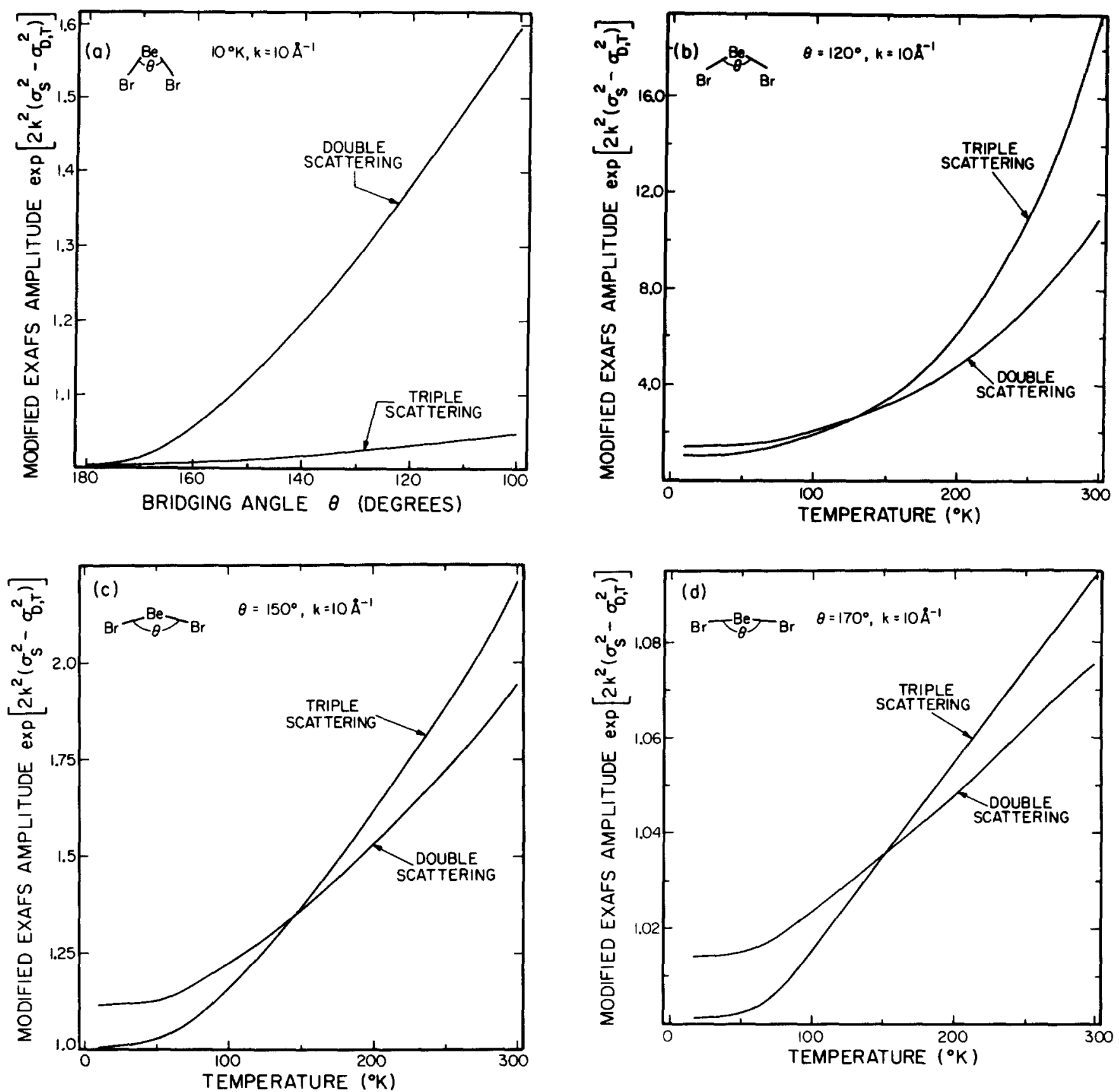

FIG. 9. Modification in the EXAFS amplitude of the double and triple scattering terms in the $\mathrm{BeBr}_{2}$ system due to the assumption that the second shell single scattering Debye-Waller factor $\left(\sigma_{S}\right)$ may be used to approximate the double $\left(\sigma_{D}\right)$ and triple $\left(\sigma_{T}\right)$ scattering Debye-Waller factors. All amplitudes were calculated at $k=10 \AA^{-1}$. (a) Modification in the amplitudes of the double and triple scattering terms at $10 \mathrm{~K}$ as a function of bridging angle $\theta$. (b) Temperature dependence at a bridging angle of $120^{\circ}$. (c) $\theta=150^{\circ}$. (d) $\theta=170^{\circ}$.

The calculations in Fig. 9, however, assume that the single scattering and multiple scattering paths contribute equally to the EXAFS. The ratio of the intrinsic amplitudes of the double and triple scattering terms is

$$
\left|2 \chi_{D}^{0} / \chi_{T}^{0}\right|=\frac{2\left(\hat{r}_{i} \cdot \hat{r}_{j}\right) r_{j} r_{i j}\left|f_{i}(\beta, k)\right|}{r_{i}\left|f_{j}(\alpha, k)\right|\left|f_{i}(\pi, k)\right|},
$$

where $\alpha$ and $\beta$ are the scattering angles at atoms $j$ and $i$ and the $T$ matrices in Eqs. (17) and (18) have been written in terms of their respective scattering amplitudes. In instances where multiple scattering is important, $120^{\circ}<\beta<180^{\circ}$ and $\left|f_{i}(\pi, k)\right| \gtrsim\left|f_{i}(\beta, k)\right|$ due to the presence of a local maximum in the scattering amplitude in the backward direction. ${ }^{17}$ For symmetric systems $r_{j}=r_{i j}$ and $r_{i}=2 r_{j}\left(\hat{r}_{i}, \hat{r}_{j}\right)$ so that the ratio is given by

$$
\left|2 \chi_{D}^{0} / \chi_{T}^{0}\right| \sim r_{j} /\left|f_{j}(\alpha, k)\right| .
$$

The triple scattering EXAFS component will dominate when the bond distance $r_{j}$ is small or when the scattering amplitude through an angle $\alpha$ is large for the intervening atom $j$. In general, the multiple scattering term which dominates is dependent on the geometry of the system and the nature of the intervening atom. If one term completely dominates the EXAFS, then the other terms may be neglected 
and Teo's approximation is always valid. The errors shown in Fig. 9 are significant only if both multiple scattering terms and the single scattering term are comparable or if one multiple scattering term and the single scattering term are comparable while the remaining multiple scattering term is small. If both multiple scattering terms are comparable in magnitude and greater than the single scattering term, then we must compare the difference in the Debye-Waller factors of the multiple scattering terms to obtain the error in the amplitude. This error is typically smaller than those shown in Fig. 9 (see Fig. 8).

A study of the temperature dependence of the DebyeWaller factors reveals that there exists a temperature where the double and triple scattering factors are equal. This crossover point occurs because the triple scattering path is less sensitive than the double scattering path to the low frequency bend mode. Since the frequency of this mode increases as the system becomes less bent the temperature of the crossover point also increases (see Fig. 8). Provided the single scattering contribution to the EXAFS is small, an analysis of data collected at this temperature, and based on Teo's assumption, is rigorous and should yield accurate results. If the normal frequencies of vibration are known and a reasonable estimate $\left( \pm 15^{\circ}\right)$ of the bridging angle exists this crossover temperature may be calculated from Eqs. (28) and (12).

Recently, Alberding and Crozier ${ }^{18}$ discussed the analysis of EXAFS data which contained a significant amount of multiple scattering. They considered a $\mu$-oxo system, $\mathrm{Fe}_{2} \mathrm{O}$, and a dihydroxy bridged system, $\mathrm{Fe}_{2}(\mathrm{OH})_{2}$. The $\mathrm{Fe}_{2} \mathrm{O}$ system is approximately symmetric with a bridging angle of $164^{\circ}$ and an $\mathrm{Fe}-\mathrm{O}$ bond length of $1.8 \AA$. Using an analysis similar to that described by Teo, ${ }^{7}$ Alberding and Crozier obtained a bridging angle of $180^{\circ}$ and an $\mathrm{Fe}-\mathrm{Fe}$ distance of $3.63 \AA$ (compared to an actual distance of $3.55 \AA$ ). These results are not surprising since Teo's analysis is not strictly valid in this instance, especially at high temperature [see Eq. (41) and Fig. 8]. A calculated bridging angle of $180^{\circ} \mathrm{might}$ be explained by the fact that the Debye-Waller factors associated with each scattering path which are constrained to be equal by the analysis, are in fact, equal only at this bridging angle. A second model analysis involving a bending mode was also proposed. This latter method obtained a better $\mathrm{Fe}-$ Fe distance and bridging angle. The residual surface, however, was broad and ill defined. For a general analysis a combination of both of these methods should be used, since in reality, even the bend mode produces a change in the $\mathrm{Fe}-\mathrm{O}$ distances and the stretching mode produces some change in the scattering angle.
In conclusion, the Debye-Waller factors in EXAFS spectra which contain a significant multiple scattering component are sensitive to the geometry of the system. The accuracy of Teo's approximation depends on which term or terms dominate the EXAFS for the system in question. In general, there will be a significant discrepancy if any two or more of the scattering paths involving the second shell atom have comparable amplitude. The magnitude of this discrepancy is smallest for systems with large bridging angles and at low temperatures. In instances where the contribution to the observed EXAFS from the single scattering path is small a temperature range exists in which the above approximate is very accurate.

\section{ACKNOWLEDGMENTS}

The authors wish to thank Dr. Folim G. Halaka for advice and many helpful discussions. National Science Foundation (Grant No. CHE12589) for their financing the research. Contribution No. 6817 from the Arthur Amos Noyes Laboratory of the Department of Chemistry, California Institute of Technology, Pasadena, California 91125.

'D. E. Sayers, E. A. Stern, and F. W. Lytle, Phys. Rev. Lett. 27, 1204 (1971).

${ }^{2}$ P. A. Lee, P. H. Citrin, P. Eisenberger, and B.M. Kincaid, Rev. Mod. Phys. 53, 769 (1981).

${ }^{3}$ V. V. Schmidt, Bull. Acad. Sci. USSR Phys. Ser. 25, 988 (1961); 27, 392 (1963).

${ }^{4}$ G. Beni and P.M. Platzman, Phys. Rev. B 14, 1514 (1976).

${ }^{5}$ R. B. Gregor and F. W. Lytle, Phys. Rev. B 20, 4902 (1979).

${ }^{6}$ E. Sevillano, H. Meuth, and J. J. Rehr, Phys. Rev. B 20, 4908 (1979).

${ }^{7}$ B. K. Teo, J. Am. Chem. Soc. 103, 3940 (1981).

${ }^{8}$ J. J. Boland, S. E. Crane, and J. D. Baldeschwieler, J. Chem. Phys. 77, 142 (1982).

${ }^{9}$ See, for example, A. A. Maradudin, G. W.Montroll, A. H. Weiss, and I. P. Ipatova, Theory of Lattice Dynamics in the Quadratic Approximation (Academic, New York, 1971).

${ }^{10}$ M. Lax, Rev. Mod. Phys. 23, 287 (1951).

"See, for example, E. B. Wilson, J. C. Dicius, and P. C. Cross, Molecular Vibrations (McGraw-Hill, New York, 1955).

${ }^{12}$ M. Born and K. Sarginson, Proc. R. Soc. London Ser. A 179, 69 (1941).

${ }^{13}$ See, for example, T. L. Hill, Introduction to Statistical Thermodynamics (Addison-Wesley, Reading, Mass., 1960).

${ }^{14}$ M. Born, Rep. Prog. Phys. 9, 294 (1943).

${ }^{15}$ See, for example, G. Hertzberg, Infrared and Raman Spectra (Van Nostrand, Princeton, 1945).

${ }^{16} \mathrm{~K}$. Nakamoto, Infrared Spectra of Inorganic and Coordination Compounds, 3rd ed. (Wiley, New York, 1970).

${ }^{17}$ P. A. Lee and J. B. Pendry, Phys. Rev. B 11, 2795 (1975).

${ }_{18}^{18}$. Alberding and E. D. Crozier, Phys. Rev. B 27, 3374 (1983).

${ }^{19}$ J. J. Boland and J. D. Baldeschwieler, J. Chem. Phys. (submitted). 